\title{
État de la recherche en éducation comparée
}

\author{
Pierre-Louis Gauthier
}

\section{OpenEdition}

Journals

Édition électronique

URL : http://journals.openedition.org/ries/1317

DOI : 10.4000/ries.1317

ISSN : 2261-4265

\section{Éditeur}

Centre international d'études pédagogiques

\section{Édition imprimée}

Date de publication : 1 septembre 2005

Pagination : 146-154

ISBN : 978-2-85420-564-0

ISSN : 1254-4590

\section{Référence électronique}

Pierre-Louis Gauthier, "État de la recherche en éducation comparée », Revue internationale d'éducation de Sèvres [En ligne], 39 | septembre 2005, mis en ligne le 17 novembre 2011, consulté le 01 mai 2019. URL : http://journals.openedition.org/ries/1317; DOI : 10.4000/ries.1317

Ce document a été généré automatiquement le 1 mai 2019.

(C) Tous droits réservés 


\title{
État de la recherche en éducation comparée
}

\author{
Pierre-Louis Gauthier
}

1 Les colloques et rencontres internationales répondent à la nécessité des échanges propres au monde scientifique qui se nourrit du dissentiment et de la confrontation. Ces manifestations offrent l'opportunité de prendre connaissance en temps réel des méthodes, des démarches et des résultats à travers les domaines de la recherche. Organisé à La Havane en octobre 2004, le congrès mondial des sociétés d'éducation comparée a pris un caractère particulier du fait, d'une part, de la place éminente que tient l'éducation à $\mathrm{Cuba}^{1}$ en dépit des pressions externes et des tensions internes que connaît actuellement ce pays et, d'autre part, des réserves et des préjugés que ce choix a pu susciter. En fait, la localisation de cette manifestation mondiale en Amérique latine a permis d'offrir une tribune aux chercheurs de ce continent souvent en déficit de liberté d'expression et faire émerger une vision nouvelle des problèmes du développement, audelà de tout exotisme et de tout préjugé occidental.

2 À partir du millier de communications présentées au congrès, on peut tenter une première analyse mettant en évidence les tendances actuelles de la recherche en éducation comparée. Tout d'abord en termes chiffrés, notons que les communications provenant de soixante-huit pays présentaient des différences sensibles suivant les continents. Qui s'en étonnera? Dresser l'état de la recherche en éducation revient quasiment à retracer l'état du monde et de ses déséquilibres.

3 On constatera une fois de plus ${ }^{2}$ l'écrasante prédominance de la recherche nordaméricaine, bien structurée dans des universités de haute qualification ${ }^{3}$. Cependant, les représentations européenne (180, communications), sud-américaine (164), asiatique (97), et africaine (52) attestent de la vigueur de la recherche sur tous les continents en dépit des difficultés économiques et politiques que connaissent maints pays participants. On remarquera également que les travaux en langue française ont retrouvé une place qu'ils avaient perdue ${ }^{4}$. On n'attendra pas de cet article une improbable présentation exhaustive 
des travaux présentés, mais plutôt quelques aspects de la recherche, les communications citées ne l'étant qu'à titre d'illustrations.

Autour du thème central du congrès "Éducation et justice sociale ", on a vu se dessiner une typologie des thèmes qui constituent la trame des grands débats dans le monde de l'éducation d'aujourd'hui.

\section{La globalisation de l'éducation}

5 Espoir pour les uns, crainte pour les autres, la globalisation de l'éducation est sinon le thème le plus souvent abordé, du moins celui qui s'est imposé comme le leitmotiv des échanges. Liée aux conséquences d'une économie libérale au développement inouï et incontrôlé, la globalisation pose à l'éducation d'innombrables interrogations. Tous les continents sont concernés, de l'Asie (Kim, Corée), (Tai, Taïwan), à l'Afrique (Chetty, Afrique du sud), à l'Amérique latine (Moreno Moreno, Brésil), à l'Amérique du nord (Smith, États-Unis), au Moyen-Orient (Cernetic, Syrie). Encore faut-il raison garder et se maintenir dans des paramètres de comparabilité acceptable. Quelles sont les fins des réformes qui s'imposent dans tous les systèmes éducatifs au nom de la nouvelle économie «digitale» (Arnove, États-Unis) ? Comment identifier les nouvelles idéologies sousjacentes (Arjmand, Suède; Randall-États-Unis; Nagata, Japon)? La globalisation se confond-elle avec l'expansion de «l'Empire » (Kachur, Canada ; Gasser, États-Unis) ? Que signifie le discours de la Banque mondiale (Steven, États-Unis)? Le passage des changements globaux à l'échelle locale révèle les distorsions régionales, voire nationales (Welch, Australie). Preston (Royaume-Uni) met bien en relief cette dialectique du local et du global et de la redéfinition de la citoyenneté qu'elle engendre face à la globalisation à la base. D'une manière générale, la globalisation absorbe et transforme le singulier en standards internationaux (Tolley, Nouvelle Zélande).

\section{La remise en cause des identités éducatives}

6 La remise en cause des identités éducatives fait l'objet de nombreuses études, le plus souvent bien contextualisées. Ainsi en va-t-il dans la Caraïbe (Quintero, Cuba), au Brésil (Streck, Brésil), en Afrique (Abdoulaye, Suisse), en Australie (Morgan et al.), en Asie (Bireda et al., Japon), en Europe (Almeida, Portugal). Les comparaisons menées au sein des sociétés rurales montrent qu'elles subissent de plein fouet et comme sans résistance possible cette vague de bouleversements prédateurs. C'est le cas pour la Chine (Liu), l'Afrique du Sud (Paterson), le Kenya (Rew), le Guatemala (Holbrock). La pauvreté générée par la mondialisation, rend inopérants les systèmes traditionnels d'éducation (Lange, Burkina Faso) et l'éducation non-formelle elle-même est victime de ces phénomènes de désagrégation sociale (Younoussi, Burkina Faso). Plus complexes encore à résoudre sont les problèmes provoqués par les migrations économiques et politiques de populations avec leur cortège d'exclusions, d'acculturations, de marginalisations, tels qu'ils se posent au Portugal (McBrien), en Chine (Wang), en Australie (Abu-Dohou et al.), en Belgique pour les ressortissants de l'ex-Union soviétique (Bruynickx et al.), au Japon pour les réfugiés vietnamiens (Hosoya, Kojima) et pour les enfants d'origine étrangère (Sonoyama), aux États-Unis pour les Mexicains (Bosque) les Asiatiques (Lew), les Haïtiens (Brown Lilly). Les minorités connaissent un sort comparable: ainsi pour les Aborigènes en Australie (Morgan, Kuteliek), les Tais aux États-Unis (Vichit, Vadakan), les populations nilotiques 
au Kenya (Rew), les Mayas au Guatemala (Holbrock), en Afrique du Sud (Mda), à Taïwan (Chen), au Laos (Fry, États-Unis), au Québec (Parédès).

7 Parce qu'elle est l'une des clés de l'ordre économique nouveau, l'éducation reste un enjeu politique dans lequel l'enseignant éprouve quelque difficulté à préserver neutralité et liberté de jugement (Koskinen, Finlande), (Mederos et al., Cuba). L'alternative entre éducation multiculturelle et discours nationaliste s'impose à lui (McAndrew, Canada ; Regnault, France).

\section{Tensions, conflits et reconstruction}

On sait que la globalisation ne met pas fin aux tensions et conflits, ni à leurs conséquences pour l'éducation. Au contraire, les inégalités sociales accroissent la «crise de l'éducation ». La violence dans l'institution (Leach, Royaume-Uni), est précédée par des symptômes maintenant bien identifiés, de l'absentéisme au décrochage ${ }^{5}$, et qui se répètent de système en système, en France (Pain, Oeuvrard, Montoya, Cellier, Hedibel, Trancart), en Afrique du sud (Muthukrishna et al.), au Royaume-Uni (Turner). De la violence dans l'institution à la violence territoriale et au terrorisme, il n'y a parfois qu'une infime distance (Nelles, Canada). Monkman (États-Unis) préconise une évaluation commune et participative pour dépasser les tensions, désormais intégrées dans l'acte éducatif. Résorber les tensions et restaurer l'éducation est devenue une tâche prioritaire et la gestion post-traumatique des conflits pose aux systèmes éducatifs de redoutables défis. Comment réconcilier et réparer tout en préservant le devoir de mémoire? (McKnight, États-Unis). Comment restaurer la solidarité sociale dans une société « fracturée »? (Cathelain, France ; Expecton, Banque mondiale ; Torres, Colombie ; Taksic, Croatie ; Chetty, Afrique du sud ; Gallagher, Irlande du nord).

La reconstruction ne peut esquiver aucun des secteurs de l'éducation mis à mal par les conflits armés (Limage, Buckland, Tawil, Unesco ; Toranzos, Argentine ; Kallaway, Afrique $\mathrm{du}$ sud). C'est le cas en Irak (Dall, Owens et al., États-Unis), au Congo (Miatelana), en Palestine (Sultana, Malte). Les situations de transition politique génèrent également tensions et conflits internes. Le contexte européen, pourtant fertile en expériences de transition, a fourni peu de recherches comparatives: Zachariev (France) traite de l'Europe centrale et orientale, Kuzma de la Pologne, Rodica de la Roumanie.

\section{Systèmes éducatifs post-coloniaux}

Dans l'élaboration de systèmes éducatifs post-coloniaux, le transfert de modèles décontextualisés constitue souvent une version «soft» du néocolonialisme, comme à Mayotte (Tupin, France) ou à La Réunion (Meynent, France). Un petit État post-colonial, Fiji, fait l'objet d'une étude de Coxon (Nouvelle-Zélande). Les contextes post-coloniaux ont donné lieu à deux panels présidés par Hickling-Hudson, présidente du congrès. Il y a là tout un champ où la recherche française est peu présente, longtemps empêtrée dans une conception néo-coloniale de l'éducation outre-mer, et fourvoyée dans des directions stériles (les institutions internationales) ou technocratiques (les indicateurs).

11 Le tumulte dû aux bouleversements de la globalisation, s'apaise lorsque s'imposent de nouveau les thèmes fondateurs de l'éducation. La recherche d'un monde éducatif en équilibre avec des sociétés fragilisées passe par une redéfinition des valeurs de 
l'éducation (Brock, Royaume-Uni). Dans cette perspective humaniste on voit fleurir les thèmes qui distinguent l'acte éducatif du dressage (Waterkamp, Allemagne), (Lin, ÉtatsUnis ; Valdès-López, Cuba ; Xing, Chine).

\section{Droit à l'éducation et apprentissage de la démocratie} (Banque mondiale) et Mulinga (Ouganda) en rappellent les fondements pour l'Afrique, Audivert Coello pour Cuba, Roque Martinez pour la Caraïbe, Aydagul pour la Turquie. L'application des droits de l'enfant est étudiée au Japon (Horio), en Europe (DàvilaEspagne), au Royaume-Uni comparé au Japon et aux États-Unis (Aspinall). Ces valeurs s'inscrivent naturellement parmi les droits humains et l'apprentissage de la démocratie (Viciedo Dominguez, Cuba ; Estevao, Portugal). La responsabilité sociale (Day, RoyaumeUni), la formation du citoyen comparée entre Cuba (Valdès Lopez), le Mexique (Alcantara Santuario), les États-Unis (Wilkinson), l'Afrique du Sud (Vaughn) et la Hongrie (Katz), en constituent les corollaires.

\section{L'éducation à l'international et à la paix}

L'éducation à l'international et à la paix dispose maintenant d'un véritable corps de doctrine dont les réalisations sont examinées en Norvège (Brock Utre), au Japon (Nagata) qui est comparé avec l'Inde (Terano, Ginsburg, États-Unis). Les nombreuses coopérations entre États renforcent cette solidarité (Villalón, Cuba).

\section{L'éducation des filles et des femmes}

Le premier corollaire du droit à l'éducation concerne l'éducation des filles et des femmes, principe qui est loin d'être respecté dans le monde. L'inégalité entre les sexes devant l'éducation subsiste: au Bénin (Klissou), au Kénya (Aniango), au Mexique (Guzman), à Taïwan (Chen), en Afrique du sud (Siyakwasi, Palm, Foster, Le Roux), en Europe (Christensen, Danemark; Psunder, Slovénie; Apak, Turquie ; Vrcej, Croatie) aux ÉtatsUnis (Jah), en particulier chez les chicanas émigrées du Mexique (Heredia). Dans le monde, quatre cent millions de femmes restent analphabètes et parmi elles, pas une seule Cubaine (Rivero Pino)!

\section{L'éducation à l'environnement et à la santé}

Devant les dangers qui menacent les équilibres sur la planète, l'éducation à l'environnement monte en puissance : Rodriguez (Cuba), Salas Gomez (Espagne), Mathias (USA) et bien d'autres ont présenté des études sur ce thème. L'éducation à la santé est l'un des volets de toute éducation moderne (Sverker, Suède). La lutte contre les grandes endémies et en premier lieu le HIV, est au premier plan et il est remarquable que le congrès ait réservé une tribune sur ce sujet aux communications émanant de pays où le problème fut longtemps nié, minimisé ou déformé (Mazibuko, Swaziland; Wang, Chine ; La Serna-Perou; Breidlid et al., Baxen, Afrique du sud). La médecine, pas plus que l'éducation n'est neutre! 


\section{L'évolution des différents ordres d'enseignement} et de l'université (Mesikammen, Finlande; Paul, France). L'universalisation des enseignements universitaires est un thème dominant (Llantada, Cuba) bien que la mobilité des étudiants relève en fait plus de conditions sociologiques et géographiques que du développement de l'enseignement supérieur (Andriamampiana et al., France). La pertinence de la formation universitaire face aux besoins des pays pauvres, face à la demande sociale et économique fait l'objet de plusieurs études (Fernandez Morales, Cuba). Monat (États-Unis) s'interroge sur la pseudo neutralité des enseignements et Zarifis (Grèce) sur le maintien des valeurs dans l'université. La sélection sous toutes ses formes est étudiée par Yates (États-Unis) et Ruchen (Taïwan).

\section{La formation des enseignants}

La convergence européenne des politiques de formation n'est-elle pas une première étape vers la globalisation (Senent Sánchez, Espagne) ? La formation des enseignants représente un enjeu capital face à la globalisation. De par ses effets retardateurs, elle risque de 
prolonger la crise de l'éducation si des réformes radicales n'interviennent pas au Mexique (Fernandez-Perez), en Ethiopie (Findley, États-Unis), en Afrique du sud (Kallaway). La redéfinition du professionnalisme se pose en termes cruciaux au Royaume-Uni (Gumus), en France et au Chili (Soetard, France), au Brésil (Ivanise, Canen), au Venezuela (Sanchez Carraño), aussi bien que dans les systèmes éducatifs en reconstruction de l'Afrique du sud (Karlsonn, Tisani) ou du Vietnam (Banik, États-Unis). L'entrée réussie dans la profession reste la clé de l'insertion dans un tissu social souvent fragilisé où les attentes des communautés sont précises (Deleigne et al., France). Des études comparent les conditions de cette insertion au Chili et en Afrique du sud (Avalos et al.), en Roumanie (Rodica et al.), en Ethiopie (Aklog, États-Unis). Les recherches montrent l'inadéquation de la formation avec la réalité scolaire (Jaime Aragon, Cuba; Hordatt Gentle, Jamaïque). Enfin, la recherche comme moyen de formation est considérée comme l'un des moteurs de développement par Barbosa Abadia (Brésil), Chirino Ramos (Cuba), Fuentes Miranda (Chili). La formation des cadres de l'éducation a trouvé place dans les travaux de Newman (Jamaïque), de Sun-Keung (Australie et Hong Kong), de Botha (Canada).

\section{La formation permanente des enseignants}

21 La formation permanente des enseignants est une préoccupation partagée par Carreño (Venezuela), Gabriel (France), Lafontaine (Canada), Costa Escarra (Cuba). Mendoza Tauler et al. (Cuba) préconisent une procédure révolutionnaire de «transformation permanente» des pratiques à la faveur de la formation continue. L'évaluation de la formation des enseignants reste cependant un secteur minoritaire de la recherche (Yoon, Corée). Dans cette réflexion concernant la formation dont on aura remarqué la densité et la diversité, les considérations socio-culturelles concernant les enseignants sont très présentes au Brésil (Canen), en Australie (Gamage et al.), au Japon (Ueyama et al.).

22 Les pratiques scolaires sont abordées par le biais du projet pédagogique mis en rapport avec la culture, l'éthique et le civisme des enseignants (Fontenelle Catrih, Brésil), leur engagement personnel (Gilmour, Afrique du Sud; Kohli, États-Unis; Chacón Artega, Cuba). Dans les sociétés où le métissage est devenu la référence culturelle, comme à Taïwan (Chiang), aux États-Unis (Stewart, Palmer), au Canada (Nicol), le rôle et la place du multiculturalisme sont dominants. Par contre, en Europe, l'eurocentrisme reste de mise (D'Sena, Royaume-Uni).

\section{L'enseignement des disciplines}

L'enseignement des disciplines fournit ample matière à la recherche. Les travaux sur les langues et leur didactique, langue maternelle et langues étrangères (Piquemal, France; Schreffer, Garente, États-Unis), sont de loin les plus fournis, de l'apprentissage précoce (Villaume, France) au bilinguisme (Holmarsdottir, Norvège). En matière de politique linguistique, les États imposent leurs propres stratégies aux systèmes éducatifs et le plus souvent la langue nationale aux dépens des langues locales et d'immigration : en Australie (Aoki, Lili, Japon), au Maroc (Tood, États-Unis), au Nigeria auprès des populations nomades (Usman, Canada), en Chine (Yang, Taïwan), au Québec (Amireault, Canada). Ces politiques linguistiques hégémoniques sont génératrices d'inégalités et la réflexion née de la comparaison, porte sur l'équilibre à obtenir entre langues dominantes et langues 
modimes. C'est à travers les légendes, les histoires, les littératures indigènes, que le patrimoine linguistique peut être sauvegardé (Archibald, Canada). Des communications ont porté sur les minorités en Israël (Kaiman), en Afrique du sud (Reagan, États-Unis), à Cuba (Beltran), aux États-Unis (Teran, États-Unis). L'approche des grandes aires culturelles et de leurs littératures font l'objet de plusieurs études comparatives, aux États-Unis (Anderson), en Europe (Ferrer, Espagne). Cette éducation à l'interculturel est également tournée vers la sauvegarde des cultures des migrants (Vega Puente, Cuba). Mais, face à la globalisation, les enseignants sont-ils préparés à relever les nouveaux défis linguistiques se demande Fox (Australie) ? La formation des enseignants à l'international ne saurait esquiver cet aspect du métier (Hernández, Cuba).

D'autres champs disciplinaires font l'objet de travaux, tout aussi prometteurs: les mathématiques (Mickelson et al., États-Unis ; Giovanelli et al., États-Unis ; Karuratua, Sri Lanka; Alvarez Perez, Cuba), l'histoire (Vickers, Royaume-Uni), les sciences sociales (Treva Turner, États-Unis), les sciences de la nature (Naidoo, Afrique du Sud), l'éducation physique et sportive (Lentillon et al., France; Brawdy, États-Unis), les nouvelles techniques de communication (Yoshumura, Japon ; Balossouka, France).

\section{Vers une pédagogie post-moderne}

Dans cette perspective élargie aux pratiques se situent les recherches sur une pédagogie post-moderne de l'éducation mondialisée dont le baccalauréat international serait la première étape concrète (Richardson, États-Unis ; Eriguchi, Japon). La pédagogie Freinet est interpellée sur la lutte contre les inégalités par Carra (France), de même que les pédagogies fondées sur l'étude du milieu et de l'environnement (Lutasik, États-Unis) et la pédagogie du projet personnel (Akosua Adare, États-Unis), (Blondeau et al., France), (Basco, France). Ainsi, après des décennies d'errance sur des pistes stériles, des chercheurs français se tournent à nouveau vers les champs des pratiques scolaires. À ce stade, on constatera que, dans une sorte de glissement épistémologique, la pédagogie comparée, est hissée au rang de science (Mendoza, Cuba). Une synthèse de la pensée pédagogique en Amérique latine a ainsi été présentée par Escanaverino et al. (Cuba), cependant que Quintero rappelait l'œuvre pédagogique de l'incontournable Jose Marti.

\section{L'évaluation de la qualité de l'éducation et des formations}

26 L'évaluation de la qualité de l'éducation et des formations ouvre, entre évaluations internationales, nationales, régionales, un champ permanent pour la comparaison (Simola, Finlande). Les résultats des évaluations PISA, leur résonance et leurs conséquences variables d'un pays à l'autre sont intéressantes à confronter (Storm, Finlande; Berge, Norvège). On compare ainsi les dispositifs nationaux d'évaluation dans les pays ibéro-américains, en Argentine (Lanza et al.), à Cuba (Bajos et al.), au Mexique (Ramírez) et parmi les pays du Mercosur (Alvarez Ruiz, Argentine). D'autres formes de contrôle sont également examinées comme l'inspection (Gutierrez, Cuba) ou l'autoévaluation (González González, Cuba). L'enseignement tertiaire n'est pas épargné par la vague de l'évaluation, au Québec (Gomes), à Taïwan (Rurnq), au Japon (Muta), en Argentine (Corengia), à Cuba (Carrasco Jiménez et al., Cuba), au Mexique (Tabasco et 
Zuniga). Ici, l'évaluation devrait également s'attacher à la relation étudiant-enseignant (Verhine, Brésil ; Giannakaki, Royaume-Uni ; Lecointe-France), de même qu'aux valeurs d'équité et d'intégration sociale dont l'enseignement supérieur devrait être porteur (Nicaise, Belgique).

\section{Les réformes}

Cette mouvance du monde éducatif incite nombre de chercheurs à comparer les réformes à travers les méthodes, les résultats obtenus (Jakubielski, Pologne), par exemple entre Russie et Chine (Boresvskaya, Russie). Ces études proposent toutes la démocratisation des procédures (Calderón, Cuba). Si les réformes liées à l'éducation tout au long de la vie semblent marquer le pas après les semi-échecs constatés en Afrique (Borg et al., Malte) et l'insuffisance des actions de l'UNESCO (Davies, Brésil), les modèles récents semblent émerger d'Asie (Sawano, Japon; Gupta, Inde ; Al Harthi, Oman).

La mise en œuvre du Processus de Bologne et l'universitarisation de l'enseignement supérieur sont à ranger parmi ces études (Suspitsin et al., États-Unis ; Charlier, Belgique ; Remedios González, Cuba).

\section{Le financement de l'éducation}

Le financement de l'éducation, et a fortiori des réformes et de l'innovation, est générateur d'inégalités profondes (Orivel, France; Paul, France; Lewin, Royaume-Uni). L'irrésistible ascension du néo-libéralisme induit les effets pervers de la marchandisation de l'éducation à travers diverses formes de privatisation, telles qu'ont pu les étudier Neridoo pour l'Afrique du Sud, Yeak pour les États-Unis, Krawczyk pour le Brésil, Srisvastavas en Inde, Oviawe en Asie centrale, Krpsoi au Kenya, Kjell à travers trois provinces du Canada, Koskinen en Finlande. L'évolution de la participation domestique aux frais d'écolage, que Wayack a étudiée au Burkina Faso et Chudgar aux États-Unis, est un précieux indicateur de ces phénomènes, de même que le développement prébendaliste (Sagnier, Argentine).

\section{La gouvernance de l'éducation}

30 La gouvernance de l'éducation, thème récurrent dans les systèmes éducatifs, implique les méthodes et les techniques de management les plus récentes. Les petits États et les régions se présentent souvent comme des laboratoires d'étude et d'expérimentation D'où l'intérêt des travaux de Bindebir (États-Unis) sur Taïwan, de Kravetz sur la province de Córdoba en Argentine, de Tan sur Singapour. L'autonomie est l'un des thèmes dominants dans le domaine (Cortez, Chili), notamment dans l'enseignement supérieur (Navarro, Mexique). L'Asie là encore est un lieu d'innovation, en Chine (Mok, Yang), au Japon (Yonesawa), en Corée du sud (Welch), à Taïwan (Chen).

31 La gouvernance est aussi le lieu d'exercice du pouvoir avec ses inévitables antagonismes, conflits et résistances (Govender, Afrique du Sud ; Evans, Jamaïque ; Correa, Brésil). Y a-til une éducation de la résistance (Perumal, Afrique du sud; Brulé, Canada ; Correa et al., Brésil)? 


\section{La méthodologie de l'éducation comparée}

Devant un tel foisonnement, la méthodologie de l'éducation comparée, longtemps négligée, trouve un regain de vigueur. Fondée sur une réflexion sur la philosophie de la comparaison (Wang, Chine; O'Dowd, Suède) et ses finalités actuelles (Rodriguez, Cuba), sur la neutralité de la science (Ardizzone, États-Unis), la recherche s'interroge sur les valeurs véhiculées par la comparaison (Waterkamp, Allemagne; Suzuki, Japon) et ses liens avec la philosophie politique (Randall, États-Unis). L'épistémologie de l'éducation comparée (Pampanini, Italie; Schriewer, Allemagne; Welch, Australie; Osborn et Newman, Royaume-Uni) implique la définition de nouveaux paradigmes et de nouvelles aires culturelles. Les méthodes de l'éducation comparée (Nichols, Royaume-Uni ; LopezPalacio, Cuba), son enseignement (Cordóba, Argentine), sa place dans la formation (Veloz, Cuba) complètent le tableau.

On remarquera que les disciplines connexes de l'éducation, telles que la psychologie (Dufays, Fontaine, Belgique; Mosselson, États-Unis; Tellez, Mexique), l'anthropologie (Prieto, González), l'histoire de l'éducation (Quddus et al., Norvège) sont peu représentées, contrairement à la sociologie qui ouvre la voie à la contextualisation des études.

La décentration hors de la sphère d'influence occidentale opérée par ce congrès d'éducation s'est révélée fructueuse et sans aucun doute féconde pour la recherche en éducation dans le monde. L'ouverture à des chercheurs souvent confinés dans leurs cantons a mis en lumière la vitalité de la recherche dans les pays d'Amérique latine. La parole rendue à des centaines de chercheurs des aires hispanophones, lusophones, francophones, voire anglophones, aura plus fait pour la liberté d'expression que bien des discours convenus. L'explicitation du droit à l'éducation, totalement mis en œuvre à Cuba, a permis de rappeler que les droits humains constituent un tout indissociable dans une démocratie.

\section{NOTES}

1. Rapport du Laboratoire latino-américain d'évaluation de la qualité de l'éducation, Unesco, Paris, juillet 2004.

2. Voir graphique.

3. P-L. Gauthier, "États-Unis : les tendances actuelles de la recherche en éducation comparée ", Revue internationale d'éducation de Sèvres, $\mathrm{n}^{\circ}$ 34, décembre 2003.

4. Actes du symposium francophone de La Havane, à paraître en octobre 2005. (afec.bureau@clubinternet.fr). Pour consulter les actes du congrès mondial de La Havane : www.wcces.hk.u

5. « Décrochages et raccrochages scolaires », Revue internationale d'éducation de Sèvres n 35, avril 2004. 
INDEX

Mots-clés : éducation comparée, recherche en éducation

\section{AUTEUR}

PIERRE-LOUIS GAUTHIER

Inspecteur d'académie (H). 